\title{
Shape Optimization of High-Speed Rail by Biomimetic
}

\author{
Chin Toong Foo ${ }^{1}$, Badrul Omar ${ }^{1, *}$, Ishkrizat Taib ${ }^{2, *}$ \\ ${ }^{1}$ Universiti Tun Hussein Onn Malaysia, Engineering Design Research Group (EDRG), 86400 Batu \\ Pahat Johor, Malaysia \\ ${ }^{2}$ Universiti Tun Hussein Onn Malaysia, Flow Analysis, Simulation, and Turbulence Research Group \\ (FAST), 86400 Batu Pahat Johor, Malaysia
}

\begin{abstract}
The Southern Corridor High-Speed Rail (HSR) has the potential to connect Kuala Lumpur and Singapore with only 90 minutes of travel time. The HSR needs to travel at minimum drag in order to promote ride comfort and environmental sustainability. Eight combinations of train head bio-models are generated from a morphological chart of three key concepts formed by biomimetic method, referring to the peregrine falcon, belted kingfisher, sailfish and dolpine. The three key concepts are the type of train nose, cross-section shape, and train nose shape. The bio-models are drawn by computer-aided drawing software and the air flow around the bio-models are simulated in order to obtain the drag coefficients in counterflow condition. The drag coefficient of each bio-models are compared and validated with published drag coefficient of Harmony CRH380A, which is 0.1971 . The bio-models with lowest drag coefficient is the bio-model with sharp-streamlined nose and round-based crosssection, which is 0.1559 . The drag coefficient of Harmony CRH380A is reduced by $20.9 \%$ if the windscreen is removed and the paraboloid nose is replaced with sharp nose. These bio-models can be a guideline for future studies in developing a more sustainable train shape in the railway industry.
\end{abstract}

\section{Introduction}

High-speed rail (HSR) is one of the rail-type transports that enables mass passengers to travel from places to places within a short time. HSR is generally defined as rail transport that could operate at a high speed, and the European Union specifically defines high-speed rail as lines specially built for train of speeds equal or greater than $250 \mathrm{~km} / \mathrm{h}$. In countries or cities with high population such as China and Japan, high-speed rail is a necessity to accommodate the movement of the citizens. [1]

Malaysian government has listed Southern Corridor HSR which has the potential to connect Kuala Lumpur and Singapore as one of the Entry Point Projects for the Economic

\footnotetext{
*Corresponding author: badrul@uthm.edu.my
} 
Transformation Programme (ETP). High-speed rails are important where demand for travel between large cities is high and will continue to increase [2]. There is a need to improve connectivity between the two countries, as currently daily traffic congestion is critical and far exceeds the capacity of the Causeway by $33 \%$, with the current infrastructure provisions [3][4]. Travelling with car is currently the most preferred mode of transportation between the two cities [5]. Traffic of the Causeway will continue to grow at a rate comparable to the Gross Domestic Product (GDP) growth of Malaysia-Singapore, at an average $3 \sim 5 \%$. Over longer term, the growth rate is expected to reach an average of $3.2 \%$ per annum from 2011 2060 with a market of 251 million passenger trips by 2060 [3][4].

From the Davis formula, the aerodynamic drag is known to be the main contributor to the overall drag force acting on the HSR, thus becoming one of the main limiting factor of the speed. The poor design of external shape of a HSR is one of the factors of high aerodynamic drag. The aerodynamic drag will cause the increment in the drag force which might reduce the speed of the train drastically. Furthermore, improper aerodynamic drag will induce loud noise in the train, increase structural vibrations, and trigger impulse pressure waves between two trains passing by each other. All these will lead to the discomfort of passengers on board. Thus, the aim of reducing aerodynamic drag is very important in designing a high-speed rail. [6]

In this paper, biomimetic method is used to optimize the shape of high-speed rail, where CRH380A is the scope of this research. The world fastest animals were used to extract the three key concepts. The efficiency of these key concepts which is the type of train nose, shape of train nose and the cross-sectional shape of the train, is compared in this paper. From these key concepts, eight bio-models of HSR are formed from a morphological chart and are compared to find the best combinations of key concepts. Finally, the shape of CRH380A is then optimized based on the best bio-models in terms of lowest drag coefficient.

\section{Aerodynamics of Train Nose}

According to Schetz [7], the nose and tail only contribute $8 \sim 13 \%$ of the aerodynamic resistance. The significance of nose and tail pressure reduction is low over a large variety of slender shapes, as long as there is no sharp edges. A poorly designed train nose will cause the train to exit a tunnel with an explosive noise because the train nose is subjected to micro-pressure wave. Micro-pressure waves are affected by train speed, train-to-tunnel area ratio, slenderness of the nose and et cetera [8].

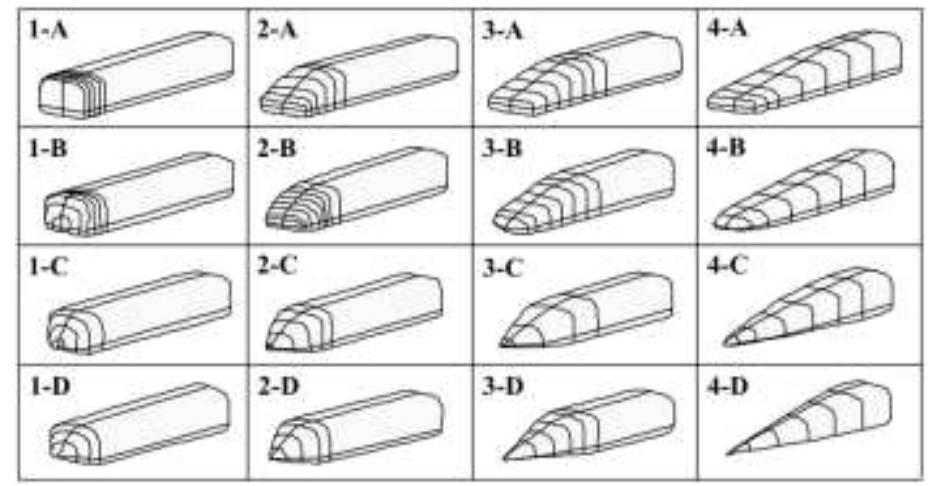

Fig. 1. Model train configuration. [9] 
Figure 1 shows a typical specimens of wind tunnel test which yields 16 results. The alphabets A, B, C, and D represents the different cross-sectional area of the nose while the numbers represents the length of change of cross-sectional area. The train head series-1 and series-A has the highest drag coefficient compared to the other series of train head. The reduction in drag coefficient is insignificant after the length-to-width ratio reaches 1.0. However, the length-to-width ratio of the train tail significantly influence the drag coefficient. The higher the length-to-width ratio, the lower the drag coefficient. [9]

The nose shape of the G7 Project of Korean Government is an ellipsoid. Lee \& Kim [8] had discovered that paraboloid nose shape is the most efficient shape compared to ellipsoid and circular cone nose shape. The paraboloid nose shape gives the lowest maximum micropressure waves. However, an optimized nose shape designed by $\mathrm{Ku}$ et. al. [10] is compared to the paraboloid nose shape of Lee at the length range of $5 \sim 15 \mathrm{~m}$. The optimal shape has a negative gradient along the length of the nose in the middle section as shown in Figure 2. The maximum micro-pressure waves of the optimal shape compared to the paraboloid shape is reduced by $18 \sim 27 \%$.

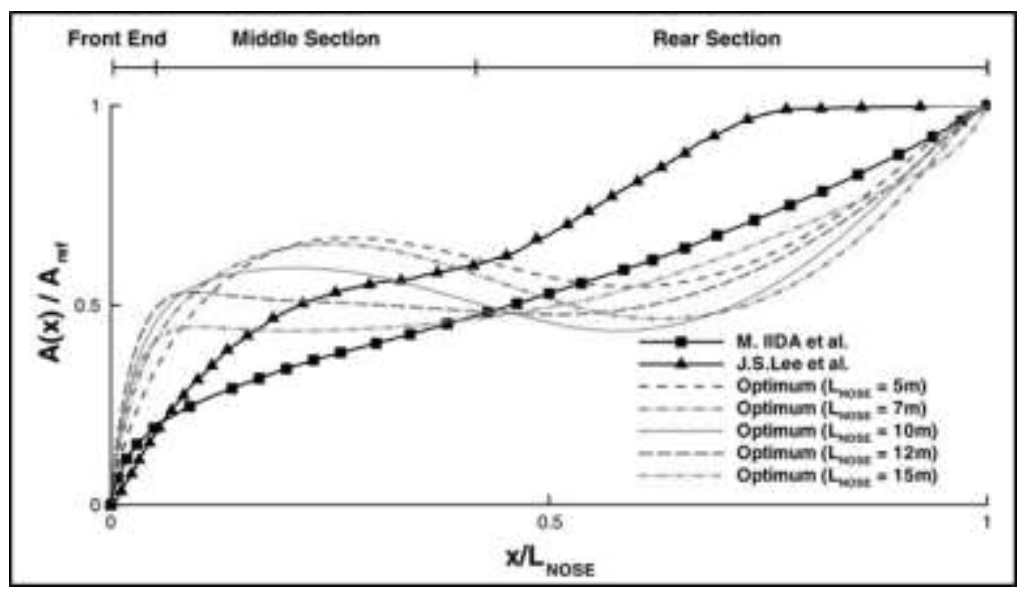

Fig. 2. Comparison of paraboloid shape (J. S. Lee) and optimal shape (Optimum). [10]

The CRH380A has eight cars with the pantographs located at the fourth-car and sixthcar. From Figure 3, we are able to know that the drag force of the fourth-car and the sixthcar has the largest drag coefficient. Relatively, the train-head and train-tail have the largest drag.

Yao et. al. [11] optimized the streamline of the head of CRH380A by using Kriging method, CFD analysis, and multi-objective optimization method. Table 1 shows the result of the force coefficient of the optimized shape and original shape. Figure 4 shows that the pressure contour of the optimized shape at the train nose is reduced and the shape of the train head is more streamlined by smoothing out the edge marked with " 1 ". 


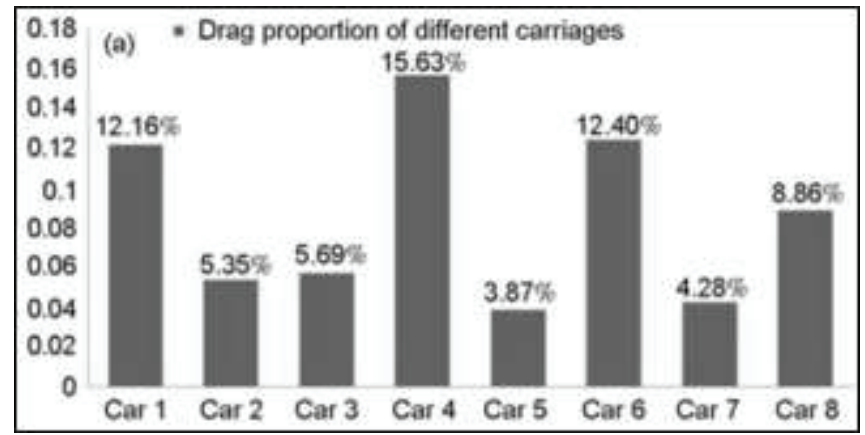

Fig. 3. Drag coefficient distribution of CRH380A. [6]

Table 1. Aerodynamic force coefficients. [11]

\begin{tabular}{|c|c|c|}
\hline Model type & Total C $_{\mathbf{D}}$ & Reduction \\
\hline Original shape & 0.1971 & \\
\hline Optimal shape & 0.1908 & $3.20 \%$ \\
\hline
\end{tabular}

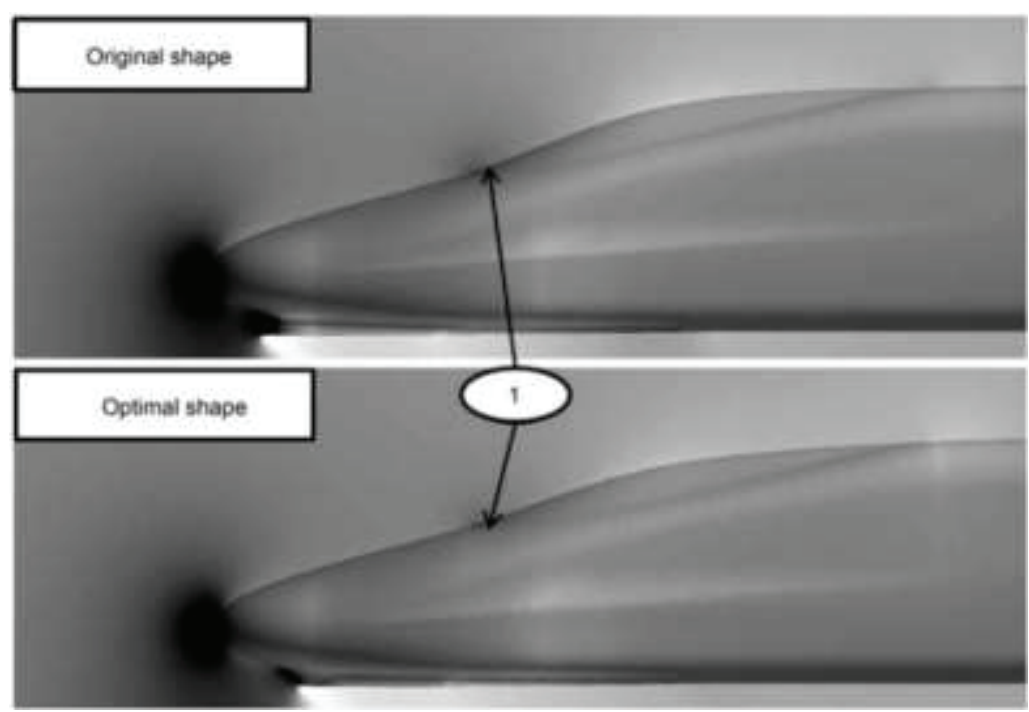

Fig. 4. Pressure contour around the streamline of the optimal shape and original shape. [11]

\section{Biomimetics}

Biomimetic is widely used in designing streamlined train nose shape [12]. As discussed in section 2.3.2, Kim \& Lee [12] developed the BTM with drag reduction by $21 \%$. The chief engineer of the Shinkansen, Eiji Nakatsu, used biomimetic to mimic the bill of a belted kingfisher as the basic shape of the Shinkansen 500 Series train head. The explosive sound caused by the impact of micro-pressure wave on the train nose as the train enter or exit a tunnel is greatly reduced $[13,14]$.

It is not the best way to achieve an optimal design by mimicking directly. Verification and validation of a solution-shape is crucial in developing bio-model. This can be done by 
evaluating and modifying every sub-features in relation to design constraints. Adapting the parametric modelling from evolutionary biology could provide a complementary methodology by which to validate and verify the original bio-model shape, allowing designers to seek a solution shape closer to optimal for each specific design challenge. Some previous effort ignored the verification and validation of a solution-shape because they were too focused on mimicking the original shape and delivering its original characteristics [12]. In order to overcome the issues of credibility of designs by biomimetic, a set of proper procedures and morphological evaluation is included in this research in order to choose the best combination of bio-model generated.

Aerodynamics and hydrodynamics characteristics of different animals are the subject of interest in this research. Head shapes of different fast-moving organisms such as birds, fishes, and eagles, are studied and observed for developing the train nose. Marine animals is included in this biomimicry perspective report. The animals chosen for this research is peregrine falcon, belted kingfisher, sailfish, and common dolphin.

An adult peregrine falcon is the fastest animal in the world that dives at a speed over $386 \mathrm{~km} / \mathrm{h}(230 \mathrm{mph})$. The subject of interest on the peregrine falcon is the shape profile of its head. A belted kingfisher could fly at a speed ranged between $58 \sim 72 \mathrm{~km} / \mathrm{h}$. However, speed is not the subject of interest. A kingfisher is able to dive into water, separating the water with its bill to capture its prey and flew back out of the water within seconds. The subject of interest on the belted kingfisher is its ability to penetrate into water with its long bill at high-speed which other birds could not. Sailfish is known as the fastest fish in the ocean. It could swim at a speed up to $110 \mathrm{~km} / \mathrm{h}$. It has highly streamlined body enabling it to swim fast. The subject of interest in the sailfish is its long mouth and streamlined body. Its fins are not considered in this research because trains do not need fins to balance while travelling on a rail track. Common dolphin could swim up to a speed of $40 \mathrm{~km} / \mathrm{h}$, which is faster than the world fastest Olympic swimmer. The subject of interest in the dolphin is its highly streamlined shape because it has strong muscles which allows it to sprint. In order for it to sprint at high-speed, streamlined is important for it. These four animals are chosen as they seems to possess the characteristics required to solve the problem of aerodynamic drag faced by high-speed rail.

The key concepts that characterizes each of these organisms also characterize the strategies adopted by each organism and could be used to extend our understanding of such strategies, increasing the chances of finding answers for the challenges proposed. These key concepts and new functions are then used to produce different combinations of train nose shape by morphological evaluation. The three key concepts obtained from the animal model test are type of nose, cross-section shape, and nose shape. Bio-models are obtained by combining different key concepts by morphological evaluation. The bio-models are then compared with the relative design of CRH380A as the benchmark of this research.

The three key concepts are first tabulated in a morphological chart, shown in Figure 5, yielding eight different combinations of bio-model train head as shown in Figure 6. Model drawings are prepared with SOLIDWORKS 2016 software based on the combinations from the morphological chart. The parameters of the bio-model train heads are scaled down to $1 / 25^{\text {th }}$ of the original size as well as the CRH380A model so that the result can be compared at a common standard.

\section{CFD Modelling}

\subsection{Fluid flow characteristics}


In this research, only two of the governing equations are used; the continuity equation:

$$
\dot{m}_{\text {in }}-\dot{m}_{\text {out }}=\iint V \cdot \vec{n} d A=0
$$

and the momentum equation:

$$
\rho \frac{\partial u}{\partial t}+\rho u \frac{\partial u}{\partial x}+\rho v \frac{\partial u}{\partial y}+\rho w \frac{\partial u}{\partial z}=\left(-\frac{\partial P}{\partial t}+\frac{\partial \sigma_{x x}}{\partial x}+\frac{\partial \tau_{y x}}{\partial y}+\frac{\partial \tau_{z x}}{\partial z}\right)+\rho g
$$

The energy equation is not used because the system of this research is adiabatic, therefore no heat energy is transferred.

\begin{tabular}{|c|c|c|}
\hline Key Concept & Option 1 & Option 2 \\
\hline Nose type & Protruding Nose & Streamlined Nose \\
\hline Cross-section & & \\
\hline & & \\
\hline & & \\
\hline & & \\
\hline & & \\
\hline & & \\
\hline & & \\
\hline & & \\
\hline & & \\
\hline
\end{tabular}

Fig. 5. Morphological chart. 


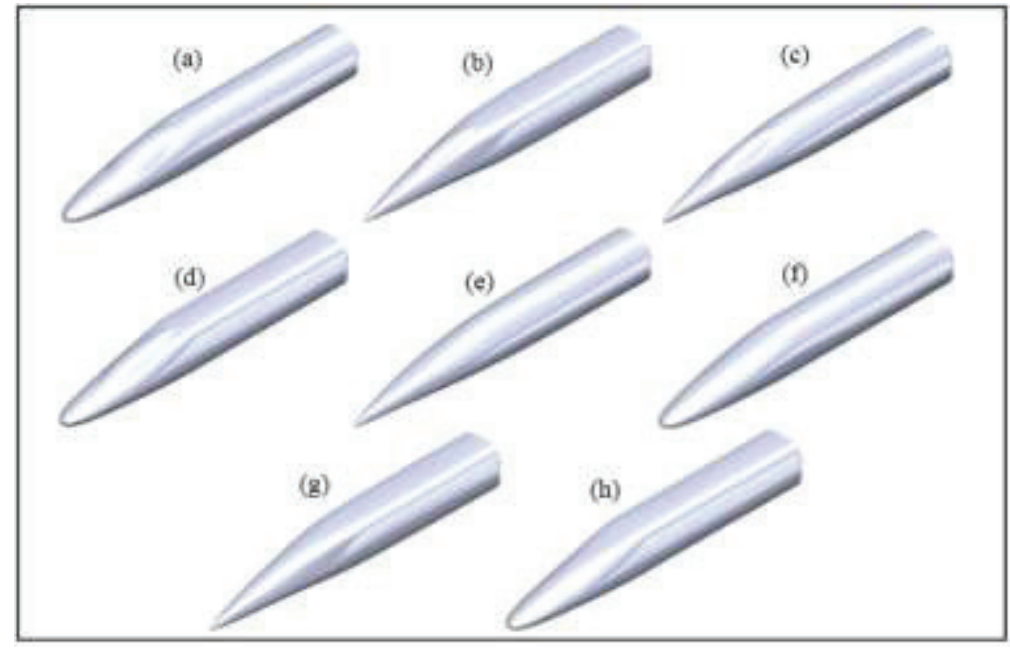

Fig. 6. Bio-models (a) 111, (b) 112, (c) 121, (d) 122, (e) 211, (f) 212, (g) 221, (h) 222.

The momentum equation is then expressed in Reynolds Averaged Navier-Stokes equation:

$$
\rho\left(\frac{\partial u^{\prime}}{\partial t}+\frac{\partial}{\partial x}\left(\bar{u}^{2}+u^{\prime 2}\right)+\frac{\partial}{\partial x}\left(\overline{u v}+u^{\prime} v^{\prime}\right)+\frac{\partial}{\partial x}\left(\bar{u} \bar{w}+u^{\prime} w^{\prime}\right)\right)=\rho g_{x}-\frac{\partial \bar{P}}{\partial x}+\mu\left(\frac{\partial^{2} \bar{u}}{\partial x^{2}}+\frac{\partial^{2} \bar{u}}{\partial y^{2}}+\frac{\partial^{2} \bar{u}}{\partial z^{2}}\right)
$$

Since the flow is inviscid and the flow is time independent, therefore the body force and the time-dependent variable are equal to zero. The RANS Equation is then reduced to:

$$
\rho\left(\frac{\partial}{\partial x}\left(\bar{u}^{2}+u^{\prime 2}\right)+\frac{\partial}{\partial x}\left(\overline{u v}+u^{\prime} v^{\prime}\right)+\frac{\partial}{\partial x}\left(\bar{u} \bar{w}+u^{\prime} w^{\prime}\right)\right)=-\frac{\partial \bar{P}}{\partial x}+\mu\left(\frac{\partial^{2} \bar{u}}{\partial x^{2}}+\frac{\partial^{2} \bar{u}}{\partial y^{2}}+\frac{\partial^{2} \bar{u}}{\partial z^{2}}\right)
$$

The turbulent model $k-\varepsilon$ realizable model is used in this research because the $k-\varepsilon$ model focuses on the mechanisms that affect the turbulent kinetic energy. It is used for far field model. This model is made up of two model equations; the transport equation for the mean turbulence kinetic energy, $k$ :

$$
\frac{\partial}{\partial t}(\rho k)+\frac{\partial}{\partial x_{i}}\left(\rho k u_{i}\right)=\frac{\partial}{\partial x_{j}}\left[\left(\mu+\frac{\mu_{t}}{\sigma_{k}}\right) \frac{\partial k}{\partial x_{j}}\right]+G_{k}+G_{b}-\rho \varepsilon-Y_{M}+S_{k}
$$

and the transport equation for the dissipation of mean kinetic energy, $\varepsilon$ that occurs in the smallest eddies:

$$
\frac{\partial}{\partial t}(\rho \varepsilon)+\frac{\partial}{\partial x_{i}}\left(\rho \varepsilon u_{i}\right)=\frac{\partial}{\partial x_{j}}\left[\left(\mu+\frac{\mu_{t}}{\sigma_{\varepsilon}}\right) \frac{\partial \varepsilon}{\partial x_{j}}\right]+C_{1} \frac{\varepsilon}{k}\left(G_{k}+C_{3} G_{b}\right)-C_{2} \rho \frac{\varepsilon^{2}}{k}+S_{\varepsilon}
$$

\subsection{CFD simulation}

The speed of airflow is $30 \mathrm{~m} / \mathrm{s}$. Only counter flow of wind is tested in this simulations. The size of the fluid domain is as shown in Figure 7. Taking the length, $L$ of the train head as a reference, the length of inflow direction is 0.5 , the total length of the domain is $4 \mathrm{~L}$, the 
width and height is $0.5 \mathrm{~L}$, and the clearance between the underbody and the rail is $0.00235 \mathrm{~L}$. In common practices the fluid domain is usually bigger almost twice the size of the fluid domain used in this research. However, this smaller domain size is used because the focus of this research is the fluid flowing near the surface of the train model and the differences of characteristics of fluid flow between the train head and train tail instead of the characteristics of the entire flow in the domain.

The model mesh is made up of 1.1 million nodes. The element size set is as default; minimum size of $5.1162 \mathrm{e}-04 \mathrm{~m}$ and maximum size of $0.102320 \mathrm{~m}$. Proximity and curvature is turned on for advanced size factor, fine relevance centre, and high smoothing. The train wall surface is covered by a five layer prism with a stretching factor of 1.2. Grid independent test is not done due to limited time and limited computing resources. Therefore, the best setting available due to constraints are chosen and verified with reference result.

Additional body sizing is also added to refine discretized parts of the model which are the nose tip, protruding head region, and the wake region. The element size for these sizing is set as $0.01 \mathrm{~m}$ and $0.02 \mathrm{~m}$ for the wake region. The face sizing for the train wall is set as $0.02 \mathrm{~m}$. Smooth transition is set as the inflation option at the train wall with transition ratio of 0.272 , maximum layer of five and growth rate of 1.2 .

The solution method used is Pressure-Based Simple Solution. The flow is steady and the velocity formation is absolute. The turbulent model used is $k-\varepsilon$ realizable model. The setting of the turbulent model is as default.

The inlet boundary layer, the density and dynamic viscosity of air at $303.5 \mathrm{~K}$ atmospheric pressure is given by $1.164 \mathrm{~kg} / \mathrm{m}^{3}$ and $1.875 \times 10^{-5} \mathrm{~kg} / \mathrm{ms}$ respectively. The fluid velocity component is $-30 \mathrm{~m} / \mathrm{s}$ at the z-direction. The turbulent intensity is $0.3 \%$, turbulent viscosity ratio is 10 , and the backflow turbulent length scale is $0.012 \mathrm{~m}$. The gauge pressure box is set as zero Pascal because the atmospheric pressure is not taken into consideration for gauge pressure. The outlet boundary conditions used is pressure outlet. The turbulent intensity is set as $5 \%$ while the remaining setting is the same as the inlet. The surrounding boundary condition is set as moving frictionless wall at a speed of $30 \mathrm{~m} / \mathrm{s}$ to the opposite of $z$-direction because the wall is a part of the flowing air. However, the rail is a stationary frictionless wall.

The turbulent kinetic energy, $k$ applies at the first order upwind while the turbulent dissipation rate, $\varepsilon$ is second order upwind for the solution method. The solver method is simple scheme. The surface area that is used to calculate the drag coefficient is the crosssectional area of the frontal side.

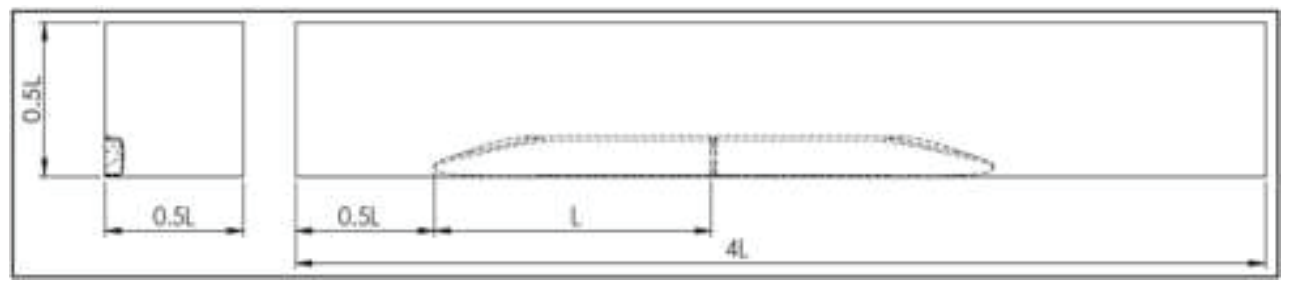

Fig. 7. Fluid domain size. 


\section{Results}

The drag coefficient of CRH380A model is 0.1973 , which is very near to the reference drag coefficient. Among the eight bio-models, bio-model 211 has the lowest drag coefficient, which is 0.1559 . The drag coefficient of each respective models are tabulated in Table 2 and compared in Figure 8. The drag coefficient for model CRH380A is 0.1973 and will be used as the benchmark in this research. The bio-models' drag coefficient obtained is lower than that of CRH380A model, ranged between $0.1559 \sim 0.1922$.

Figure 9 and Figure 10 are the pressure distribution along CRH380A model and biomodel 211. The pressure at the nose tip of CRH380A model is almost doubled of that of bio-model 211. The occurrence of high pressure region at the train head of CRH380A model is also more frequent compared to bio-model 211.

The drag coefficient of bio-models with protruding nose is higher because the occurrence of stagnation point is more due to the presence of windscreen. These high pressure regions around the windscreen that are shown in Figure 11 for model 111 is not found in model 211 and any other bio-models without windscreen. These stagnation points in the head increased the total pressure of the head which leaves the tail with a relatively lower pressure coefficient, therefore increasing the drag.

Table 2. Drag coefficient result.

\begin{tabular}{|c|c|c|c|}
\hline Train model & \multicolumn{2}{|c|}{ Published $C_{D}$} & Simulated $C_{D}$ \\
\hline CRH380A & \multicolumn{2}{|c|}{0.1971} & 0.1973 \\
\hline Bio-model & $\mathrm{C}_{\mathrm{D}}$ & Bio-model & $\mathrm{C}_{\mathrm{D}}$ \\
\hline 111 & 0.1582 & 211 & 0.1559 \\
\hline 112 & 0.1922 & 212 & 0.1826 \\
\hline 121 & 0.1655 & 221 & 0.1651 \\
\hline 122 & 0.1845 & 222 & 0.1785 \\
\hline
\end{tabular}




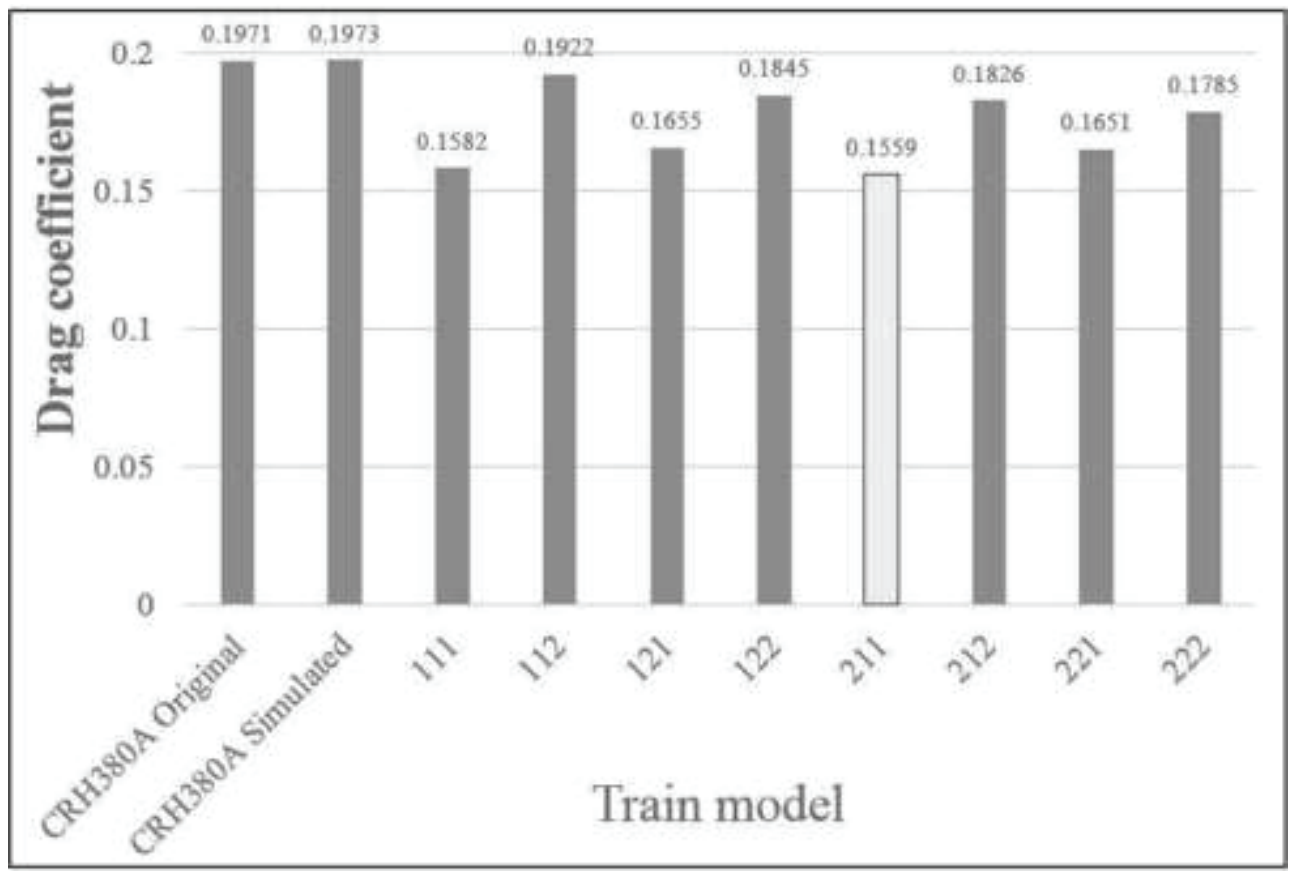

Fig. 8. Drag coefficient of different train models.

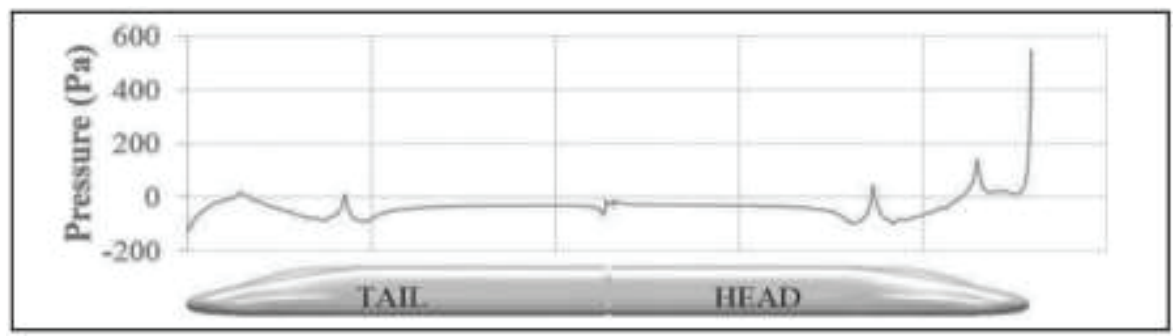

Fig. 9: Pressure distribution of Harmony CRH380A.

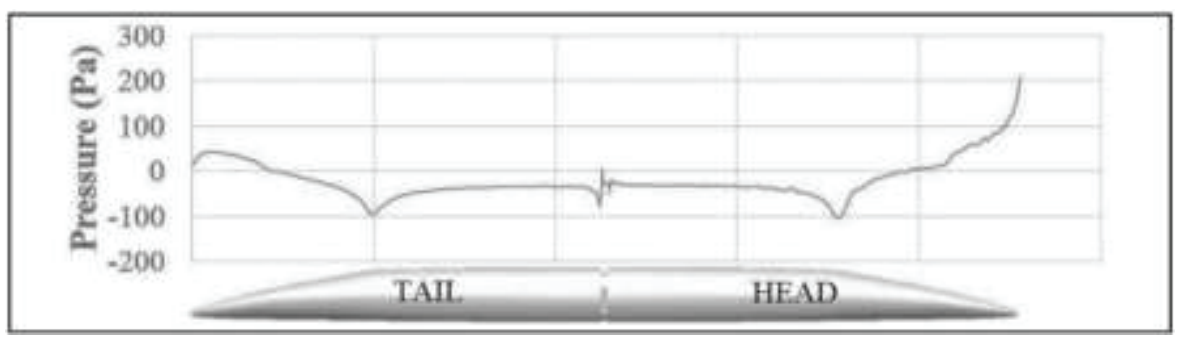

Fig. 10. Pressure distribution of Bio-model 211.

The drag coefficient of the HSR is not dependent to the cross-section shape of the train body, neither round-based (prefix 1) nor square-based (prefix 2). Figure 12 shows the comparison of drag coefficient for different groups of bio-models; round-based crosssection and square-based cross-section. The drag coefficient of 111 and 211 is lower than that of 121 and 221 by $5 \%$ while the drag coefficient of 112 and 212 is higher than that of 122 and 222 by $3 \%$. 
The drag coefficient for rounded nose shape models are higher because the area of stagnation point for rounded nose shape is larger and the impact is greater compared to that of sharp nose shape as shown in Figure 13. The maximum pressure at the nose tip of model 211 is $209 \mathrm{~Pa}$. However, the maximum pressure at the nose tip of model 212 is $555 \mathrm{~Pa}$. There is a huge difference in pressure between the two types of nose shape. Therefore, sharp nose shape is more efficient than rounded nose shape.

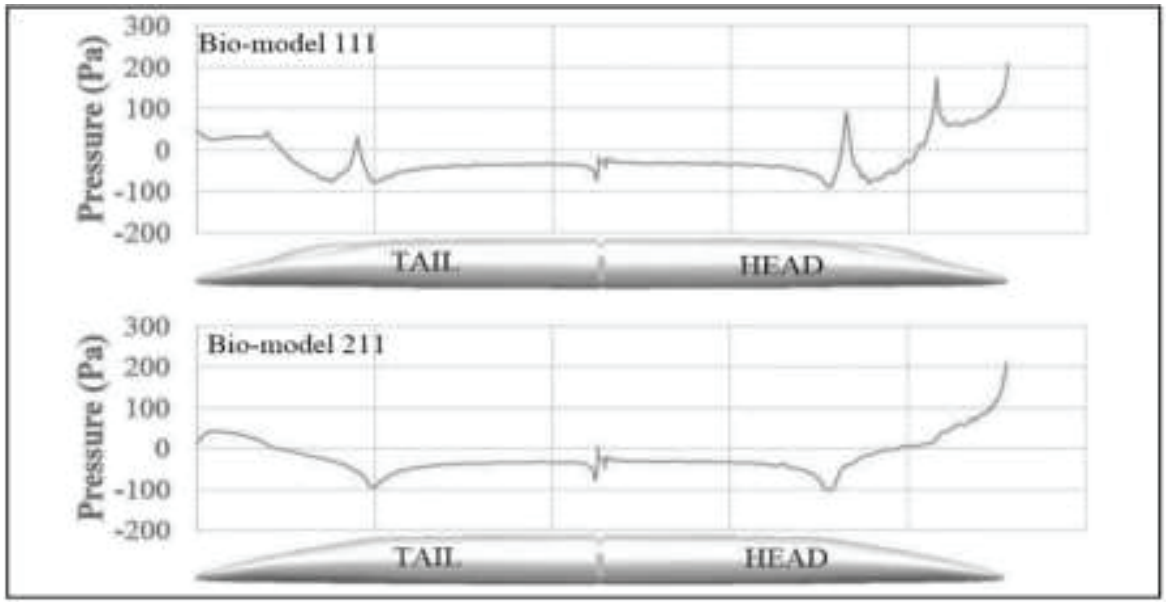

Fig. 11. Pressure distribution comparison of different nose type.

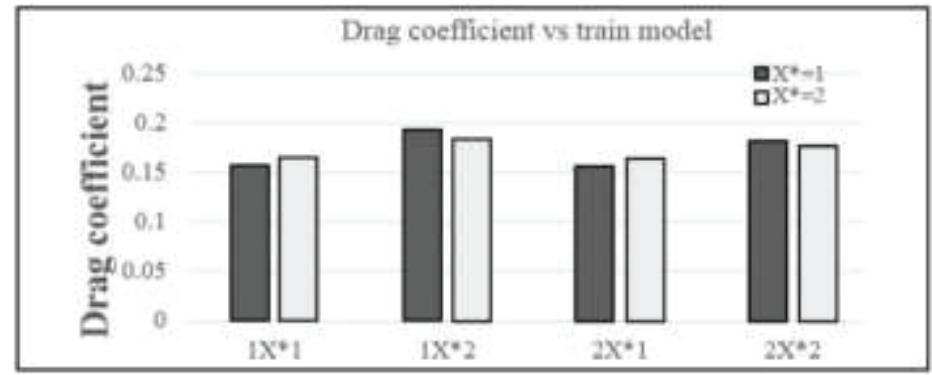

Fig. 12. Drag coefficient of train models with round-based and square-based cross-section.

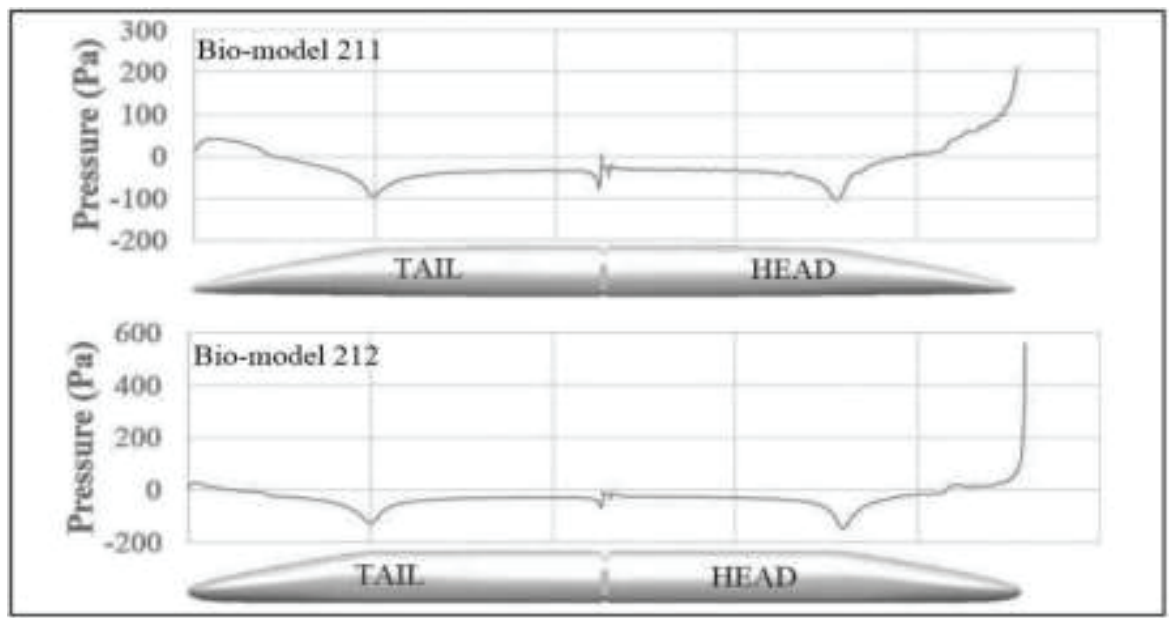

Fig. 13. Pressure distribution comparison of different nose shape. 


\section{Conclusion}

By comparing eight bio-models, it is found that bio-model 211, Figure 6 (e), is the best model with a drag coefficient of 0.1559. Bio-model 211 has sharp-streamlined nose and round-based cross-section. Though the cross-section shape doesn't affect the drag force much. However, the simulated result shows that bio-model 211 is the model with lowest drag coefficient. Train heads are best without the dome-shape windscreen in order to be more streamlined, because the windscreen will increase the frequency of micro-pressure waves at the train head. Sharp train nose is best in reduction of micro-pressure waves because the area of stagnation point is smaller.

By comparing the drag coefficient of the generated bio-models with CRH380A, it is found that all of the bio-models have lower drag coefficient. The model of CRH380A resembles bio-model 112 and 122 because both of these two models have round-protruding nose. In fact, the morphological chart of this researched is a basic guideline for building any train head model because what differs one HSR's shape from another is the shape of the nose, cross-section shape, and the presence of the windscreen. The drag coefficient of biomodel 112 and 122 is nearest to that of CRH380A, which is 0.1922 and 0.1845 respectively while the drag coefficient of CRH380A is 0.1971 .

Since CRH380 resembles bio-model 112 and 122, the optimization criteria should be the key concepts used to build bio-model 211 where the windscreen of CRH380A should be removed and be made streamlined in order to reduce the micro-pressure waves at the train head. The original nose shape of CRH380A is paraboloid. But if the nose shape is replaced by sharp nose shape, the micro-pressure waves at the train nose can be reduced dramatically. By implementing such modifications on the current model of CRH380A, the new model is expected to have a drag coefficient of 0.1559 , which is the drag coefficient of bio-model 211, with a percentage reduction of $20.9 \%$.

\section{Acknowledgement}

This paper was partly funded by the Centre for Graduate Studies UTHM and Postgraduate Research Grant (GPPS/U799). The authors are thankful to Universiti Tun Hussein Onn Malaysia (UTHM) for providing facilities support.

\section{References}

1. UIC, The High-speed Definition of the European Union, Retrieved March 26, 2016, from http://www.uic.org/highspeed (2012).

2. T. Gourvish, The High Speed Rail Revolution: History and Prospects, High Speed Two Ltd, United Kingdom. United Kingdom: High Speed Two Ltd, (2010).

3. MyHSR Corp, Kuala Lumpur - Singapore High Speed Rail Project. Retrieved March 26, 2016, (2015).

4. SPAD, Suruhanjaya Pengangkutan Awam Darat, High Speed Rail. Retrieved March 26, 2016, from http://www.spad.gov.my/projects/high-speed-rail Links, (2014).

5. H. H. Ling, A Comparison Study on the Carbon Footprint between the Proposed Kuala Lumpur - Singapore High Speed Rail and Other Transportation Modes. Universiti Tun Hussein Onn Malaysia, (2016).

6. G. Yang, D. Guo, S. Yao, \& C. Liu, Aerodynamic Design for China New High-Speed Trains. Science China Technological Sciences, 55(7), 1923-1928, (2012). 
7. Y. C., Rho, J. H., Yun, S. H., Kwak, M. H., Kim, K. H., Kwon, H. Bin, \& Lee, D. H. Optimal Cross-Sectional Area Distribution of a High-Speed Train Nose to Minimize the Tunnel Micro-Pressure Wave. Structural and Multidisciplinary Optimization, 42(6), 965-976, (2010).

8. J. Lee \& J. Kim, Approximate Optimization of High-Speed Train Nose Shape for Reducing Micropressure Wave. Structural and Multidisciplinary Optimization, 35(1), 79-87, (2008).

9. R. S. Raghunathan, H. D. Kim, \& T. Setoguchi, Aerodynamics of High-Speed Railway Train. Progress in Aerospace Sciences (Vol. 38), (2002)

10. Y. C. Ku, J. H. Rho, S. H. Yun, M. H. Kwak, K. H. Kim, H. B. Kwon, \& D. H. Lee, Optimal Cross-Sectional Area Distribution of a High-Speed Train Nose to Minimize the Tunnel Micro-Pressure Wave. Structural and Multidisciplinary Optimization, 42(6), 965-976, (2010).

11. S. Yao, D. Guo, Z. Sun, G. Yang, \& D. Chen, Multi-Objective Optimization of the Streamlined Head of High-Speed Trains Based on the Kriging Model. Science China Technological Sciences, 55(12), (2012).

12. S. J. Kim \& J. H. Lee, Parametric Shape Modification and Application in a Morphological Biomimetic Design. Advanced Engineering Informatics, 29(1), 76-86, (2015).

13. S. J. Kim \& J. H. Lee, How Biomimetic Approach Enlarges Morphological Solution Space in A Streamlined High-Speed Train Design? Proceedings of the 16th SIGraDi Conference, 538-542, (2012).

14. A. Deshpande, A. L. Goh, A. Goossens \& S. Javdani, Biomimicry for Coastal Ecofcities: Towards a Carbon Neutra Dover, UK. (R. A. Shenoi, P. A. Wilson, \& S. S. Bennett, Eds.) (Vol. 4). Southhampton, England: University of Southampton, (2013). 